Revue d'histoire de l'Amérique française

BHS REVUE D.HISTOIRE DE L'AMÉRIQUE FRANÇAISE

\title{
La nouvelle européenne et la presse québécoise d'expression française (1866-1871)
}

\section{Pierre-Louis Lapointe}

Volume 28, numéro 4, mars 1975

URI : https://id.erudit.org/iderudit/303393ar

DOI : https://doi.org/10.7202/303393ar

Aller au sommaire du numéro

Éditeur(s)

Institut d'histoire de l'Amérique française

ISSN

0035-2357 (imprimé)

1492-1383 (numérique)

Découvrir la revue

Citer cet article

Lapointe, P.-L. (1975). La nouvelle européenne et la presse québécoise d'expression française (1866-1871). Revue d'histoire de l'Amérique française, 28(4), 517-537. https://doi.org/10.7202/303393ar d'utilisation que vous pouvez consulter en ligne.

https://apropos.erudit.org/fr/usagers/politique-dutilisation/ 


\title{
LA NOUVELLE EUROPÉENNE ET LA PRESSE QUÉBÉCOISE D'EXPRESSION FRANÇAISE (1866-1871)
}

\author{
Pierre-Louis Lapointe \\ Agent de recherche en histoire \\ Société d'aménagement de \\ l'Outaouais \\ Hull
}

A l'occasion de recherches sur les réactions de la presse québécoise d'expression française au cours de la guerre austroprussienne et de la guerre franco-prussienne *, il nous est apparu nécessaire d'éclairer le fonctionnement de l'entreprise de presse au Québec à cette époque. En effet, l'attitude de la presse était conditionnée dans une large mesure par des facteurs inhérents à ce type de communication. Dans cette optique, nous nous sommes attardé à étudier les sources et l'acheminement de la nouvelle européenne ainsi que l'accès de nos journaux à cette nouvelle, parce que ces facteurs déterminaient en partie la quantité et la qualité de l'information européenne qui parvenait au public québécois.

La reproduction, pour ne pas dire le plagiat, est un phénomène universel à cette époque et la presse québécoise en fait grand usage. ${ }^{1}$ Elle pille systématiquement les imprimés qui lui tombent sous la main, pour remplir des colonnes dont le contenu, sans cet apport déterminant, se résumerait à quelques dépêches (pour la plupart tirées de journaux plus fortunés capables de se payer un abonnement au service de dépêches de la Montreal Telegraph Company, elle-même abonnée au câble administré par l'Associated Press) et à de rares correspondances en provenance d'Europe. C'est ce qui explique l'importance que les journaux québécois attachent à l'arrivée d'une "malle d'Europe" ou d'un vapeur apportant une "liasse de journaux"."

* A ce sujet, voir notre thèse de maîtrise, "La presse québécoise d'expression française face au problème franco-prussien, 1866-1871" (Université d'Ottawa, 1974).

1 P.-L. Lapointe, "La presse québécoise d'expression française à l'heure de la Confédération", Asticou, nos 10-11 (1973): 15-19. — Le Pays, 9 juin 1866.

2 Le Courrier du Canada, 9 novembre 1870.

[ 517 ]

RHAF, vol. 28, no 4 (mars 1975) 
La reproduction, dont l'importance ne fait aucun doute quant à la nouvelle locale ou nationale, se révèle encore beaucoup plus généralisée en ce qui concerne l'information européenne. Elle est d'autant plus fréquente que la nouvelle en provenance d'Europe est difficile d'accès et qu'elle est généralement lente à parvenir aux journaux du Québec. Les abonnements aux journaux européens sont trop coûteux pour nos entreprises de presse. ${ }^{3}$ L'installation d'un câble, à l'été 1866 , accélère certes la transmission de la nouvelle européenne, mais cette nouvelle ne parvient qu'aux journaux qui sont abonnés au service de l'Associated Press.

\section{A. SOURCES DE LA NOUVELLE EUROPÉENNE :}

\section{Les imprimés}

Les journaux québécois d'expression française s'adressent surtout aux sources imprimées lorsqu'ils désirent couvrir l'actualité européenne, et l'imprimé par excellence pour fins de reproduction, c'est le journal et, plus particulièrement, le journal francophone.

Les critères qui président au choix des journaux sont multiples mais l'on choisit de préférence ceux qui sont d'une idéologie apparentée à la leur. En effet, les principaux journalistes québécois entretiennent des liens avec leurs confrères de la presse française et ils sont conscients des tendances idéologiques des principaux journaux francophones de France, de Belgique, de Suisse et des Etats-Unis.

La plupart des journalistes les plus connus ont voyagé en Europe et certains d'entre eux ont même collaboré à la rédaction de journaux français. C'est ce que révèle Le Pays du 13 septembre 1870, à l'occasion d'une polémique sur la république française, en soulignant la position avantageuse du Constitutionnel des Trois-Rivières, de L'Evénement et du Courrier de St-Hyacinthe, dont les rédacteurs, qui défendent le système républicain, connaissent la France pour y avoir séjourné. ${ }^{4}$ Cette expérience leur permet de donner une image plus fidèle de la réalité européenne.

3 P.-L. Lapointe, op. cit., 21-31.

4 Ainsi peut-on lire dans Le Pays, 13 septembre 1870: "Le rédacteur actuel du Courrier de St-Hyacinthe a lui aussi voyagé en Europe, habité Paris et collaboré ainsi que le rédacteur du Constitutionnel de Trois-Rivières, au Journal de Paris." Les rédacteurs de ces journaux étaient alors Oscar Dunn et Elzéar Gérin. Voir A. Beaulieu et J. Hamelin, Les journaux du Québec, de 1767 à 1964 (Québec, P.U.L., 1965), 242 et 273. 
Les journaux québécois s'intéressent toujours à la vie des journaux et des journalistes européens. Certains s'abonnent au mensuel L'Imprimerie dont Le Canadien se fait probablement l'agent québécois. ${ }^{5}$ Le Canadien publie des appréciations sur la liberté de presse en France. ${ }^{6}$ Des journaux québécois reproduisent des listes de journaux français et britanniques accompagnés de leur tirage respectif. ${ }^{7}$ D'autres se montrent méfiants à l'endroit de certains journaux européens qui, insinuent-ils, font partie de l'organisation de presse de Napoléon III ou de celle de Bismark. Le Canadien du 7 avril 1869 nous parle du "gouvernement français et (de) la presse officieuse" tandis que Le Courrier $d u$ Canada du 28 septembre 1866 nous éclaire sur "deux journaux dans les intérêts de M. de Bismark (sic), le Norddeutsche Zeitung et la Kölnische Z eitung". "Quelques jours auparavant, Le Courrier du Canada publie une correspondance de L'Emancipation belge qui accuse L'International de Londres d'être une "feuille très dévouée au cabinet des Tuileries et quasi-officieuse". ${ }^{9}$ La Minerve admet également que L'International est un "organe semi-officiel du gouvernement français à Londres". ${ }^{10}$ Cela ne l'empêche pas, semble-t-il, d'échanger ou d'être abonné à ce même journal. Presque tous les journaux québécois sont agents pour le compte de journaux ou de périodiques français. Nous avons déjà vu que Le Canadien annonce L'Imprimerie. ${ }^{11} L a$ Minerve vend des abonnements pour Le Monde et pour L'Univers. ${ }^{12}$ Le Courrier $d u$ Canada fait valoir l'avantage de s'abonner à La Revue commerciale et maritime de Marseille par l'entremise de Léger Brousseau, propriétaire du Courrier du Canada.. ${ }^{13}$

Ces faits montrent jusqu'à quel point la presse québécoise est informée sur le monde journalistique européen. Les journalistes québécois peuvent donc assez facilement écarter les sources imprimées qui ne correspondent pas aux grandes lignes de leur propre position idéologique. Aussi l'origine des reproductions

${ }^{5}$ Le Canadien, 4 janvier 1867. On y offre un abonnement à ce mensuel dont on vante les mérites.

${ }^{6}$ Le Canadien, 8 avril et 10 mai 1867.

7 Le Pays, 13 février 1866; La Minerve, 29 décembre 1866.

8 Pour une présentation relativement complète de l'organisation de presse de Bismark, voir la thèse manuscrite de doctorat de Robert $\mathrm{H}$. Keyserlingk, "Bismark and the Press: The Example of the National-Liberals 1871-1884" (School of Slavonic and East European Studies, University of London, 1965).

9 Le Courrier du Canada, 14 septembre 1866.

${ }_{10}$ La Minerve, 14 décembre 1866.

11 Le Canadien, 4 janvier 1867.

12 La Minerve, 29 mars 1866 et 9 mai 1867.

13 Le Courrier du Canada, 8 février 1867. 
TABLEAU No 1

Principales sources de la nouvelle européenne

Dans Le Canadien (Québec)

\begin{tabular}{llll} 
Tendance & 1866 & $1870-1$ & Total \\
\hline
\end{tabular}

(A) Reproductions étrangères connues:

(B) Reproductions:

(1) de La France

(2) du Courrier des Etats-Unis (N.Y.)

(3) de La Gazette de France

(4) de La Liberté

(5) du Times (Londres)

(6) de L'Union de Paris

Dans Le Courrier du Canada (Québec)

(A) Reproductions étrangères connues:

$\begin{array}{lrrr}\text { Impérialiste } & 23 & 1 & 24 \\ \text { Conservateur } & 7 & 9 & 16\end{array}$

(B) Reproductions:

(1) de L'Union de Paris

(2) de L'Univers

(3) de L'Emancipation belge

(4) du Monde

(5) de L'International

(6) du Courrier des Etats-Unis

$\begin{array}{llll}\text { Légitimiste } & 8 & 0 & 8\end{array}$

Libéral

Libéral

Légitimiste

$\begin{array}{lll}3 & 3 & 6 \\ 3 & 1 & 6 \\ 2 & 0 & 2\end{array}$

$\begin{array}{lrrr} & 101 & 54 & 155 \\ \text { Légitimiste } & 44 & 0 & 44 \\ \text { Ultramontain } & 0 & 20 & 20 \\ \text { Conservateur } & 17 & 0 & 17 \\ \text { Ultramontain } & 9 & 1 & 10 \\ \text { Impérialiste } & 6 & 0 & 6 \\ \text { Conservateur } & 0 & 5 & 5\end{array}$

Dans Le Journal des Trois-Rivières (Trois-Rivières)

(A) Reproductions étrangères connues:

(B) Reproductions:

(1) de L'Univers

(2) du Monde

(3) de L'Union de Paris

$\begin{array}{lrrr} & 44 & 42 & 86 \\ \text { Ultramontain } & 0 & 35 & 35 \\ \text { Ultramontain } & 13 & 1 & 14 \\ \text { Légitimiste } & 5 & 0 & 5\end{array}$

Dans La Minerve (Montréal)

(A) Reproductions étrangères connues:

$\begin{array}{lrrr} & 8 & 17 & 25 \\ \text { Ultramontain } & 5 & 2 & 7 \\ \text { Conservateur } & 0 & 2 & 2\end{array}$

(1) du Monde

(2) du Courrier des Etats-Unis

Conservateur

$\begin{array}{lrrr} & 22 & 22 & 44 \\ \text { Conservateur } & 5 & 4 & 9 \\ \text { Pro-Autrichien } & 3 & 2 & 5 \\ \text { Libéral } & 4 & 1 & 5\end{array}$

(A) Reproductions étrangères connues:

(B) Reproductions:

(1) du Courrier des Etats-Unis

(2) du Mémorial diplomatique

(3) de La Liberté

Libéral

Dans Le Pays (Montréal)

(A) Reproductions étrangères connues:

(B) Reproductions:

(1) du Siècle

(2) de L'Opinion nationale

(3) de La Liberté

(4) du Messager franco-américain

(5) du Figaro

$\begin{array}{llll}\text { Républicain } & 0 & \mathbf{4} & \mathbf{4} \\ \text { Libéral } & \mathbf{0} & \mathbf{4} & \mathbf{4} \\ \text { Libéral } & \mathbf{1} & \mathbf{1} & \mathbf{2} \\ \text { Démocratique } & 0 & \mathbf{1} & \mathbf{1} \\ \text { Inclassable } & \mathbf{0} & \mathbf{2} & \mathbf{2}\end{array}$

Remarques: Ces données quantitatives ont été puisées à même l'échantillon utilisé lors de notre étude. Ont été compilées les reproductions traitant:

(a) de la crise nationale allemande de 1866 (1er avril 1866 au 30 septembre 1866 )

(b) de la guerre franco-prussienne (1er juillet 1870 au 30 septembre 1870 et du 1 er mars 1871 au 31 mai 1871 ).

Seules ont été retenues les reproductions dont nous connaissions l'origine. Notez l'impact de la variable géographique. Les journaux de Québec ont recours de façon beaucoup plus générale à la reproduction (Voir totaux). Il serait intéressant d'en tenter une analyse.

Notez la correspondance qu'il y a entre la tendance idéologique du journal québécois et celle des sources qu'il reproduit.

Les tendances des journaux sont celles qui sont données dans: Claude Bellanger (Editeur), Histoire générale de la presse française, Tome II (1815-1871), (Paris, P.U.F., 1969). 
nous permet-elle de découvrir l'idéologie de chacun des six journaux québécois que nous avons choisis comme étant les plus révélateurs des diverses tendances. ${ }^{14}$ On comprend qu'Arthur Buies, un contemporain, n'ait que sarcasmes pour ces "reproductions étrangères puisées invariablement aux même sources, choisies dans un même et unique ordre d'idées". ${ }^{15}$ A l'exception du Pays, la majorité de nos journaux s'abreuvent à des sources conservatrices, légitimistes et ultramontaines. La place qu'y occupent des journaux comme Le Monde, L'Union de Paris et L'Univers est imposante. ${ }^{16}$

La presse québécoise a également accès à la nouvelle européenne par ricochet. Elle peut reproduire des journaux canadiens d'expression française ou d'expression anglaise et des journaux américains qui reproduisent eux-mêmes des journaux européens. Il est pratiquement impossible de savoir jusqu'à quel point les journaux québécois puisent les uns dans les autres ou traduisent les journaux canadiens d'expression anglaise. Nous connaissons cependant l'importance du Courrier des Etats-Unis, du Messager franco-américain, du Meschacébée de Louisiane, du Courrier de San Francisco, etc. dans la presse québécoise. C'est Le Courrier des Etats-Unis qui tient le premier rang parmi ces journaux, dominant même en importance les journaux américains de langue anglaise. Le Courrier est d'allégeance conservatrice et, selon $L e$ Pays, il serait subventionné par le gouvernement canadien. ${ }^{17}$ Il est impossible de vérifier le bien-fondé de cette insinuation, même si l'on sait que P.-J.-O. Chauveau y commença sa carrière littéraire et politique comme correspondant régulier de 1840 à $1851 . .^{18}$ La presse franco-américaine prime celle de tout pays étranger à l'exception de la France, ainsi que le montre notre "Tableau analytique des sources de la nouvelle européenne selon le pays d'origine". ${ }^{19}$

Ce tableau met aussi en évidence la prédominance des sources françaises et francophones. A elles seules, les reproductions de journaux français représentent $67 \%$ de tout ce qui a trait aux nouvelles européennes et au problème franco-prussien. De plus,

\footnotetext{
péenne".

14 Voir le tableau no 1: "Principales sources de la nouvelle euro15 Arthur Buies, "La presse canadienne-française", Conférences (Québec, 1875) : 5 . péenne".

16 Voir le tableau no 1: "Principales sources de la nouvelle euro-

${ }_{17}$ Le Pays, 19 et 22 janvier 1867 ; 12 février 1867.

18 Bulletin des recherches historiques, I, 4, (avril 1895) : 60.

19 Voir le tableau no 2.
} 
TABLEAU No 2

Tableau analytique des sources de la nouvelle européenne selon le pays d'origine Les journaux reproduits par période d'analyse et par journal québécois Données brutes

\begin{tabular}{|c|c|c|c|c|c|c|c|c|c|c|c|c|c|c|c|c|c|c|c|c|}
\hline \multirow[t]{2}{*}{ Journaux reproduits } & \multicolumn{6}{|c|}{ 1er avril $1866-30$ septembre 1865} & \multicolumn{6}{|c|}{$\begin{array}{l}\text { 1er juillet } 1870-30 \text { septembre } 1870 \\
\text { ler mars } 1871-31 \text { mai } 1871\end{array}$} & \multicolumn{6}{|c|}{ TO TAU X } & \multirow[b]{2}{*}{ En semble } & \multirow{2}{*}{$\begin{array}{c}\text { Total de } \\
\text { l'ensemble } \\
\text { en \% }\end{array}$} \\
\hline & C. & C.C. & J.T-R. & M. & Ordre & Pays & C. & C.C. & J.T-R. & M. & Ordre & Pays & c. & C.C. & J.T-R. & M. & Ordre & Pays & & \\
\hline Journaux français & 52 & 67 & 29 & 7 & 13 & 2 & 29 & 41 & 41 & 13 & 10 & 22 & 81 & 108 & 70 & 20 & 23 & 24 & 326 & $67 \%$ \\
\hline $\begin{array}{l}\text { Journaux américains } \\
\text { (Total) }\end{array}$ & 7 & - & 3 & - & 5 & - & 14 & 8 & - & 2 & 10 & 2 & 21 & 8 & 3 & 2 & 15 & 2 & 51 & $10.5 \%$ \\
\hline $\begin{array}{l}\text { Journaux américains } \\
\text { (francophones) }\end{array}$ & 7 & - & 3 & - & 5 & - & 11 & 5 & - & 2 & 7 & 2 & 18 & 5 & 3 & 2 & 12 & 2 & 42 & $8.6 \%$ \\
\hline $\begin{array}{l}\text { Journaux germano- } \\
\text { prussiens }\end{array}$ & 3 & 3 & 6 & - & 4 & - & 4 & 2 & - & - & - & 3 & 7 & 5 & 6 & - & 4 & 3 & 25 & $5 \%$ \\
\hline $\begin{array}{l}\text { Journaux belges } \\
\text { (francophones) }\end{array}$ & 2 & 17 & 2 & - & - & 1 & 1 & - & - & - & - & - & 3 & 17 & 2 & - & - & 1 & 23 & $4.6 \%$ \\
\hline $\begin{array}{l}\text { Journaux britanniques } \\
\text { (Total) }\end{array}$ & 13 & 7 & 1 & 1 & - & - & 3 & - & - & 1 & 2 & 6 & 16 & 7 & 1 & 2 & 2 & 6 & 34 & $7 \%$ \\
\hline $\begin{array}{l}\text { Journaux britanniques } \\
\text { (francophones) }\end{array}$ & 1 & 6 & - & - & - & - & 一 & - & - & - & 1 & - & 1 & 6 & - & - & 1 & - & 8 & $1.6 \%$ \\
\hline Journaux autrichiens & 4 & 6 & 2 & 一 & - & - & - & - & - & - & - & - & 4 & 6 & 2 & - & - & - & 12 & $2.5 \%$ \\
\hline Journaux russes & 3 & 1 & - & - & - & - & 一 & - & 一 & - & - & - & 3 & 1 & 一 & - & - & - & 4 & $0.8 \%$ \\
\hline Journaux italiens & & & & & & & 一 & 1 & 1 & - & - & - & 3 & 1 & 1 & - & - & - & 5 & $1.2 \%$ \\
\hline Autres & 1 & - & 1 & - & - & - & 2 & 2 & - & 1 & - & - & 3 & 2 & 1 & 1 & - & - & 7 & $1.5 \%$ \\
\hline $\begin{array}{l}\text { Total - Journaux } \\
\text { francophones }\end{array}$ & 62 & 90 & 34 & 7 & 18 & 3 & 41 & 46 & 41 & 15 & 18 & 24 & 103 & 137 & 75 & 22 & 36 & 27 & 399 & $82 \%$ \\
\hline Grand Total & 88 & 101 & 44 & 8 & 22 & 3 & 53 & 54 & 42 & 17 & 22 & 33 & 141 & 155 & 86 & 25 & 44 & 36 & 487 & $100 \%$ \\
\hline
\end{tabular}

REMARQUE: Ces données ont été compilées à partir de l'échantillon que nous avons utilisé lors de notre étude de La Presse québécoise d'expression française face au problème franco-prissien, $1866-1871$. 
les reproductions de toutes sources francophones (c'est-à-dire d'origine française, belge, franco-américaine et franco-britannique) accaparent plus de $82 \%$ de l'ensemble. A qui se demanderait pourquoi une telle importance est accordée à des sources d'expression française, nous pouvons fournir deux explications: 1. il est beaucoup plus simple de reproduire un texte de langue française que de consacrer du temps à de coûteuses traductions et $d$ 'y mobiliser un personnel déjà très restreint; 2 . il existe une grande méfiance à l'égard de l'information d'origine anglo-américaine et protestante.

\section{Les dépêches}

En plus des sources imprimées, la presse québécoise se nourrit des dépêches. C'est pourquoi les journalistes accueillent avec enthousiasme le câble transatlantique, en juillet 1866. Mais leur enthousiasme est suivi, dans la presse conservatrice et ultramontaine tout au moins, de propos violents qui jettent du discrédit sur ce nouveau moyen de communication, inauguré au moment où le conflit austro-prussien provoquait un état de crise européenne qui ne pouvait laisser les esprits indifférents.

En temps normal, l'usage du câble transatlantique ne pose aucun problème au journaliste québécois de langue française. Il accélère tout simplement l'acheminement de la nouvelle. Le journaliste, que rien ne presse, continue à privilégier l'analyse, et le journal publie les dépêches sans leur accorder une place de choix dans la présentation. Il les reproduit en caractères très petits et donne la priorité aux extraits de journaux.

En temps de crise, cependant, la précipitation des événements rend la dépêche prioritaire car elle seule peut suivre d'heure en heure le déroulement des opérations militaires et des tractations diplomatiques. Le journaliste québécois est alors en plein désarroi, n'ayant plus le temps d'analyser. Ses hypothèses s'écroulent comme un château de cartes à chaque nouvelle dépêche et les imprimés qui lui parviennent trop tard ne lui sont d'aucun secours. Surtout que des six journaux québécois, rares sont ceux qui peuvent se payer un abonnement au service de dépêches de l'Associated Press. La plupart se voient obligés de reproduire les dépêches de la veille ou du matin, déjà publiées dans les journaux plus fortunés du Québec (anglophones pour la plupart). Pendant le plus fort de la guerre franco-prussienne (juillet 1870 à avril 1871), les journaux se sentent obligés d'assurer à leurs lecteurs un meilleur service de nouvelles. Ils s'abonnent donc au service 
de dépêches de l'Associated Press. L'importance que prend le conflit pour les Québécois de langue française explique cet empressement, mais, une fois qu'il est terminé, on laisse tomber l'abonnement. En plus d'avoir très mauvaise opinion du service de dépêches, on n'apprécie pas le monopole anglo-américain sur le système de transmission transatlantique. On lui attribue les dépêches contradictoires, obscures et subjectives et on voit dans les mauvaises nouvelles une conspiration des anglo-protestants et de leurs alliés prussiens.

Ainsi, au cours de la guerre austro-prussienne, Le Courrier $d u$ Canada se refuse de croire à la défaite de Sadowa, car, affirmet-il, il n'a reçu que "la version prussienne" et "le télégraphe prussien a, sous le rapport de la véracité, une réputation qui n'est pas précisément à l'abri de tout reproche". ${ }^{20}$ Le Canadien enchaîne dans la même veine: pour lui, le bulletin télégraphique reçu par l'Hermann et "rédigé dans un évident esprit d'hostilité contre la France et l'Autriche" fourmille de "contradictions et d'absurdités". ${ }^{21}$ Le Courrier du Canada se méfie du "télégraphe continental" (Kontinental Telegrafen) qui, "suivant sa louable habitude, a exagéré considérablement au profit de la Prusse". ${ }^{22}$ Le Canadien revient à la charge de façon plus explicite: il blâme l'agent londonien du New York Herald, pour le choix des dépêches faussées qui sont envoyées à l'Associated Press à New York. ${ }^{23}$. Le Courrier du Canada trouve que les bulletins télégraphiques venant de Londres sont "plus ou moins dignes de foi ... étant sous le contrôle d'une seule puissance". ${ }^{24}$ Ce même journal juge que le câble ne donne pas assez de nouvelles de Rome et du Vatican:

... ce silence du télégraphe nous fait l'effet d'une conspiration ourdie dans le but d'isoler le plus possible les catholiques du Pape en les privant des nouvelles qu'ils attendent avec tant d'anxiété de la Ville Eternelle. ${ }^{25}$

Le Pays prend la défense de l'Associated Press et tourne en ridicule ces attaques en lançant un défi à ceux qui partagent l'opinion du Courrier du Canada:

... organisez-vous, formez une société, ayez un agent et vous recevrez les nouvelles apprêtées à votre goût. Qu'une agence religieuse soit la contre-partie de l'agence des libre-penseurs. ${ }^{26}$

20 Le Courrier du Canada, 18 juillet 1866.

21 Le Canadien, 23 juillet 1866.

22 Le Courrier du Canada, 27 juillet 1866.

23 Le Canadien, 17, 20, 22 août et 5 septembre 1866.

24 Le Courrier du Canada, 26 septembre 1866.

25 Ibid., 4 février 1867.

26 Le Pays, 29 septembre 1866. 
Le Canadien revient à la charge en accusant l'Associated Press d'être responsable de dépêches télégraphiques "fabriquées à New York". ${ }^{27}$ Pour ce journal, le télégraphe transatlantique a "complètement dénaturé le discours prononcé par l'empereur à l'ouverture des Chambres françaises". ${ }^{28}$ L'Ordre fait la même constatation et se félicite "d'avoir attendu le texte officiel pour le publier". ${ }^{29}$ Le Canadien et Le Courrier $d u$ Canada sont tous deux méfiants face aux nouvelles qui viennent du Mexique par l'entremise des Américains. On refuse de croire à la proclamation de l'empereur Maximillien. C'est une nouvelle "fabriquée par nos voisins ... en vue de jouer à la hausse et à la baisse commerciale", dénonce Le Canadien..$^{30}$ C'est un "canard américain", affirme Le Courrier du Canada. ${ }^{31}$ Le Canadien se montre très dur à l'endroit "des impresarios de la Presse Américaine" en soulignant qu'il " $n$ 'y a vraiment rien de si dégoûtant que ce système menteur et absurde de nos aimables voisins". ${ }^{32}$ Et cette attitude hostile ne fait que s'amplifier au cours de la guerre franco-prussienne.

Pour la presse québécoise d'expression française, la guerre franco-prussienne prend des allures de crise de conscience nationale. Dès le début de la guerre, La Minerve met ses lecteurs en garde contre les dépêches pro-prussiennes de l'Associated Press. ${ }^{33}$ L'Ordre fait le même genre de remarque contre l'agent de l'Associated Press à Londres:

C'est à Londres, la plupart du temps, que se confectionnent ces dépêches fantaisistes ainsi que les commentaires qui les accompagnent. Il est regrettable que les nouvelles soient concentrées en cette ville pour être transmises par l'agent de la presse associée. Tant qu'il en sera ainsi ce ne sera qu'incertitudes, que chaos. ${ }^{34}$

Le lendemain, Le Canadien fait une sortie contre les nouvelles "absurdes" qui viennent d'Europe et il ajoute qu'il devrait " $y$ avoir des limites aux fabrications". ${ }^{35}$ Ce journal n'aime pas que les "ennemis de la France s'en donnent à cœur joie". ${ }^{36}$ Comme

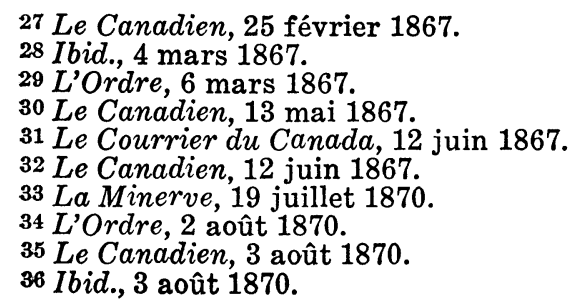


toute la presse québécoise, il refuse de croire aux défaites françaises annoncées par le service de l'Associated Press. Un correspondant de l'Ordre, qui signe "Un jeune citoyen", suggère la création d'une "Association de la Presse Canadienne, en vue d'avoir des dépêches télégraphiques pour nos journaux et à eux propres". ${ }^{37}$ Le correspondant trouve que les dépêches qui sont reçues sont trop partiales:

(Elles) ... sont entachées d'une grande partialité pour la Prusse et ont toutes une odeur Prussienne. Le correspondant de la Presse Associée est un allemand, ce qui expliquerait tout ... nous sommes à la merci des journaux de New-York, nous sommes des copistes simplement. ${ }^{38}$

La Minerve chante le même refrain: les dépêches sont obscures, partiales et contradictoires. Ce journal ministériel trouve que les rédacteurs des dépêches sont conseillés par l'ignorance et la haine:

... Que les journaux anglais, américains ou haut-canadiens, mus par une haine commune contre la France se le tiennent pour dit. Jamais une seule puissance n'a pu triompher de la France. ${ }^{39}$

Une telle confiance dans la puissance de l'ancienne mère patrie témoigne de la profondeur des sentiments que les journalistes québécois de langue française éprouvent envers la France. Le choc de la défaite sera aussi violent que ce sentiment était profond. La presse tout entière refuse de croire à la défaite de la première nation d'Europe. Même Le Pays, ordinairement si bien disposé à l'égard de l'Associated Press, se permet de dire à ses lecteurs que les nouvelles sont "de provenance américaine, et que cette voie... (est) sujette à une grande partialité". ${ }^{40}$ Ce journal met en doute "les dépêches de Berlin qui annoncent de nouvelles victoires prussiennes"41 et lorsque les nouvelles télégraphiques se font rares, il en conclut qu'elles sont défavorables à la Prusse: "A travers le mutisme du câble, on sent percer cependant nous ne savons quel souffle favorable aux armes françaises." ${ }^{2}$ Pour ne pas être en reste, La Minerve avoue savoir "de source certaine que la Prusse télégraphie ses nouvelles vraies ou fausses à des

37 L'Ordre, 11 août 1870.

38 Ibid., 11 août 1870.

39 La Minerve, 12 août et 24 août 1870.

40 Le Pays, 16 août 1870.

41 Ibid., 24 août 1870.

42 Ibid., 27 août 1870. 
organes payés par elle aux Etats-Unis"..43 L'Ordre refuse de croire aux "cancans télégraphiques au moyen desquels la Prusse cherche à se donner tous les avantages sur les Français" et il ne peut gober les "gasconnades prussiennes" qui arrivent par voie "de Prusse, de Londres et des Etats-Unis". ${ }^{44}$ Le Canadien se montre aussi confiant dans la supériorité des armes françaises que méfiant à l'égard des dépêches qui passent par Londres et New-York. Il apostrophe les responsables du service de dépêches:

Les fabricants des dépêches télégraphiques contre la France, vont s'apercevoir que les victoires ne se gagnent pas du bout de leurs doigts et que la France ne saurait être noyée et brûlée dans l'acide sulphurique de leurs batteries galvaniques. ${ }^{45}$

Le Courrier $d u$ Canada devient exaspéré: le désir de voir se confirmer une victoire française l'amène même à nier toutes les dépêches à l'exception d'une seule. Un simple doute jeté sur la défaite de MacMahon est transformé en victoire française. ${ }^{46}$ Le lendemain, L'Ordre refuse de croire à la défaite de Sédan et il préfère accréditer la rumeur selon laquelle le roi Guillaume "a perdu la tête et qu'on le renvoie à Berlin". ${ }^{47}$ Et il faudra attendre le surlendemain pour que l'ensemble de la presse québécoise d'expression française se rende à la triste réalité.

\section{Les correspondances}

Les correspondances constituent une troisième source pour la nouvelle mais cette source, statistiquement, n'a que peu d'importance. Des correspondances plus ou moins "régulières" sont expédiées à quelques rares journaux québécois par des amis qui sont en voyage ou par des journalistes européens agissant pour le compte de la presse québécoise. Ces correspondances sont fièrement mises en évidence dans la présentation du journal. (De même en est-il aujourd'hui des entreprises de presses capables de se payer des correspondants dans un certain nombre de capitales.) On les coiffe du titre ronflant "Correspondance particulière" ou "Correspondance spéciale" afin d'attirer l'attention du lecteur.

43 La Minerve, 29 août 1870.

44 L'Ordre, 29 août 1870.

45 Le Canadien, 2 septembre 1870.

46 Le Courrier du Canada, 2 septembre 1870.

47 L'Ordre, 3 septembre 1870. 


\section{B. ACCES À LA NOUVELLE EUROPEENNE :}

\section{Les imprimés}

La presse québécoise d'expression française a surtout accès à la nouvelle européenne par l'entremise des journaux européens qui parviennent aux journalistes de différentes façons.

Emmanuel Blain de St-Aubin avoue que "plusieurs journaux parisiens échangent maintenant avec les nôtres". ${ }^{48}$ Mais le plus souvent il est pratiquement impossible de déterminer s'il s'agit d'un abonnement ou d'un échange. Nous tenons que L'Ordre échange avec Le Mémorial diplomatique par la révélation qui en est faite à l'occasion d'une querelle entre L'Ordre et La Minerve au sujet d'un article rédigé dans Le Mémorial par Paul Boutet. ${ }^{49}$ Nous savons aussi que Le Courrier du Canada recoit une "très forte liasse de L'Univers, par abonnement ou par échange". ${ }^{50}$ Rien d'étonnant à ce que L'International reproduise une chronique de "Carle Tom" et L'Economiste français, un article du Canadien. ${ }^{51}$ Il y a probablement échange entre ces journaux.

\section{Les dépêches}

Les journaux québécois de langue française reproduisent ou traduisent les dépêches de la même façon qu'ils le font pour des extraits de journaux ou de périodiques. Ils ne se soucient que rarement de l'origine du bulletin de nouvelles télégraphiques. Leurs ciseaux taillent allègrement dans les journaux qui sont abonnés à des services de dépêches. Mais cette pratique est beaucoup plus répandue pour les dépêches qui traitent des affaires européennes, car l'abonnement au service de dépêches transatlantiques (acheminées par câble) est très coûteux et ne peut être défrayé que par l'entreprise de presse de grande taille, ce qui n'est évidemment pas le cas de la majorité des journaux québécois d'expression française.

L'accès à la dépêche est donc lié de près à la nature de la nouvelle. Les bulletins de nouvelles locales, régionales et natio-

48 Emmanuel Blain de St-Aubin, "Passé, présent et avenir probable de la langue française au Canada", dans Le Journal des Trois-Rivières, 22 janvier 1867.

49 L'Ordre, 26 et 29 avril 1867: La Minerve, 27 avril 1867.

50 Le Courrier du Canada, du 3 mars 1871, faisant état de la réception de 26 numéros de L'Univers.

51L'Ordre, 19 décembre 1866; La Minerve, 6 février 1867. 
nales sont plus facilement accessibles. Il semble que les six journaux québécois étudiés soient abonnés à ce genre de service qu'assurent les compagnies de télégraphe, dont les employés recueillent et rassemblent les nouvelles télégraphiées à la centrale, puis les résument en bulletins qui sont finalement livrés aux journaux du réseau. Cette activité qui, aujourd'hui, relève en exclusivité des agences de presse, se greffait alors tout simplement sur les activités régulières des compagnies de télégraphe. N'étant pas la principale préoccupation de ces compagnies, ce service de cueillette et de distribution était mal organisé. ${ }^{62}$ La Montreal Telegraph Company, qui avait absorbé presque toutes ses rivales, distribuait ses bulletins de nouvelles à un grand nombre de journaux. Pour sa part, Le Pays préférait encourager un concurrent, Le Télégraphe du Peuple. ${ }^{53}$ Quoi qu'il en soit, ce service ne semblait pas très dispendieux, car nos journaux font état d'abonnements à l'une ou l'autre de ces deux compagnies.

Il n'en est pas de même cependant pour les nouvelles télégraphiées d'Europe. Seuls, semble-t-il, La Minerve et Le Courrier $d u$ Canada sont abonnés, et de facon régulière, à ce service de dépêches transatlantiques, ${ }^{54}$ contrôlé par l'Associated Press et assuré au Canada par l'entremise de la Montreal Telegraph Company.

Les journaux québécois qui bénéficient de ce service se plaignent constamment du pillage effectué dans leurs colonnes de dépêches par les journaux qui n'y sont pas abonnés. Ainsi, La Minerve veut que les droits de reproduction soient réservés aux seuls abonnés de l'Associated Press; elle déplore la "dilapidation dont elle est l'objet par les autres journaux, qui ne paient rien pour les dépêches, mais qui les reproduisent", et elle se moque de L'Ordre et du Pays qui n'y sont pas abonnés. ${ }^{55}$ En 1870 , dans le même esprit, L'Ordre accuse Le Nouveau-Monde de faire des "réclames ébouriffantes" lorsqu'il se présente comme le "seul journal français de Montréal qui publie les dépêches d'Europe en leur entier". 56

52 M.E. Nichols, The Story of the Canadian Press (Toronto, Ryerson Press, 1948), 6.

${ }_{53}$ Le Pays, janvier et mars 1871.

$54 \mathrm{Ce}$ qui peut s'expliquer par le fait que ces deux journaux ( $L a M i$ nerve et Le Courrier du Canada), étant liés au pouvoir politique, disposaient de moyens financiers leur permettant de défrayer le coût de ce service de dépêches.

${ }_{55}$ La Minerve, 25 juillet 1866 et 19 février 1867.

56 L'Ordre, 20 et 21 juillet 1870. 
Toutefois, les journaux ne se contentent pas de faire seulement des critiques négatives. La Minerve loue Le Journal des Trois-Rivières qui "recevra désormais les dépêches de la presse associée"; mais il semble que ce soit plutôt le Constitutionnel des Trois-Rivières qui ait pris l'initiative de "cette coûteuse décision".57 En fait, Le Journal des Trois-Rivières offre ce service au grand public du 21 juillet 1870 au 6 mars 1871. Ces dépêches coûtent de $\$ 20.00$ à $\$ 25.00$ par semaine. Des abonnements à cette édition spéciale du journal sont offerts au coût de "trente sous par semaine, invariablement payables d'avance (sic)" afin de défrayer les dépenses encourues. ${ }^{58}$ Le Franco-Canadien emboîte le pas au Journal des Trois-Rivières et s'abonne au service offert par la Montreal Telegraph Company, à l'instar des grands journaux de Montréal. ${ }^{59}$

\section{Les correspondances}

Les rares correspondances publiées dans les journaux québécois que nous avons étudiés ne nous donnent que peu de renseignements sur l'accessibilité de ce mode de communication. Nous ne savons pratiquement rien des correspondants qui les rédigent. S'agit-il de Québécois en voyage d'étude, comme dans le cas d'Arthur Buies? Nous connaissons, par exemple, le correspondant du Pays pour 1866: c'est Albert Delpit qui signe Pitter Max. ${ }^{60}$ Nous devinons que le correspondant pour ce journal, en 1870, est de nationalité française, car il est appelé sous les drapeaux. ${ }^{61}$ Nous ignorons qui sont ceux de La Minerve ou de L'Ordre, mais cela importe peu puisqu'en dernière analyse, ces correspondants sont sans rapport avec le genre d'accès à la nouvelle européenne qui prédomine.

\section{ACHEMINEMENT DE LA NOUVELLE EUROPÉENNE:}

\section{Les imprimés}

La plus grande partie des nouvelles européennes sont acheminées par bateau. Presque tous les journaux européens, ainsi que les correspondances parviennent au Québec par l'entremise

${ }^{57}$ La Minerve, 23 juillet 1866.

58 Le Journal des Trois-Rivières, 21 et 25 juillet $1870 ; 6$ mars 1871.

59 Le Pays, 8 septembre 1870.

60 Le Pays, 17 juillet 1866 .

61 Ibid., 8 septembre 1870. 
de la poste. Les délais de livraison sont en moyenne de douze à quinze jours. ${ }^{62}$ Ces délais sont généralement un peu plus longs en hiver.

La presse québécoise réclame unanimement, à cette époque, une amélioration du service postal reliant la France au Canada. La Minerve trouve qu'un seul courrier par semaine ne peut suffire; elle désirerait que "la ligne de paquebots français établie entre Brest et New-York" puisse servir à la correspondance entre le Canada et la France. ${ }^{63}$ L'Ordre déplore l'inconvénient "irrémédiable de l'irrégularité des malles transatlantiques".64 Aussi est-ce dans le but d'y obvier et pour épargner "des abonnements coûteux aux revues périodiques françaises", que ce journal suggère l'abonnement à L'Echo de la France, périodique qui reproduit des textes littéraires et scientifiques puisés à même les périodiques français. ${ }^{65}$

Il arrive occasionnellement que les journaux québécois aient accès aux journaux européens par l'entremise de voyageurs revenant d'Europe. Le Canadien signale un envoi par Adolphe Hamel, de Paris, par "l'intermédiaire de M. P. Shee arrivé à Québec ce matin" d'une "Liasse de journaux européens". ${ }^{66}$ Le Courrier $d u$ Canada remercie un certain "monsieur E.B. d'Outaouais" du don d'un numéro du Journal de Rennes, "organe de la catholique Bretagne". ${ }^{67}$

\section{Les dépêches}

Les compagnies de télégraphe et les agences de nouvelles épuisent leur ingéniosité à hâter l'acheminement des nouvelles européennes. ${ }^{68}$ L'Associated Press fait guetter les bateaux "aux approches de Terre-Neuve, de la Nouvelle-Ecosse et du Massachusetts; et ce n'est que de Saint John, de Halifax, ou de Boston que ses agents lui télégraphient les nouvelles extraites des courriers d'Europe" ${ }^{69}$ Cette agence organise la transmission, à par-

62 Pierre Frédérix, Un siècle de chasse aux nouvelles (Paris, Flammarion, 1959), 80.

63 Cité dans Le Journal des Trois-Rivières, 18 juin 1867.

64 L'Ordre, 7 janvier 1867.

65 Ibid., 7 janvier 1867.

66 Le Canadien, 8 mars 1871.

67 Le Courrier du Canada, 15 mai 1867.

68 Pour un traitement adéquat des agences de nouvelles, voir: Pierre Frédérix, op. cit., 6-10 et 60-106; M. E. Nichols, op. cit., 5-8; Richard A. Schwartzlose, "Harbor News Association: The Formal Origin of AP.", Journalism Quarterly, XLV (été 1968) : 253-260.

69 Pierre Frédérix, op. cit., 80. 


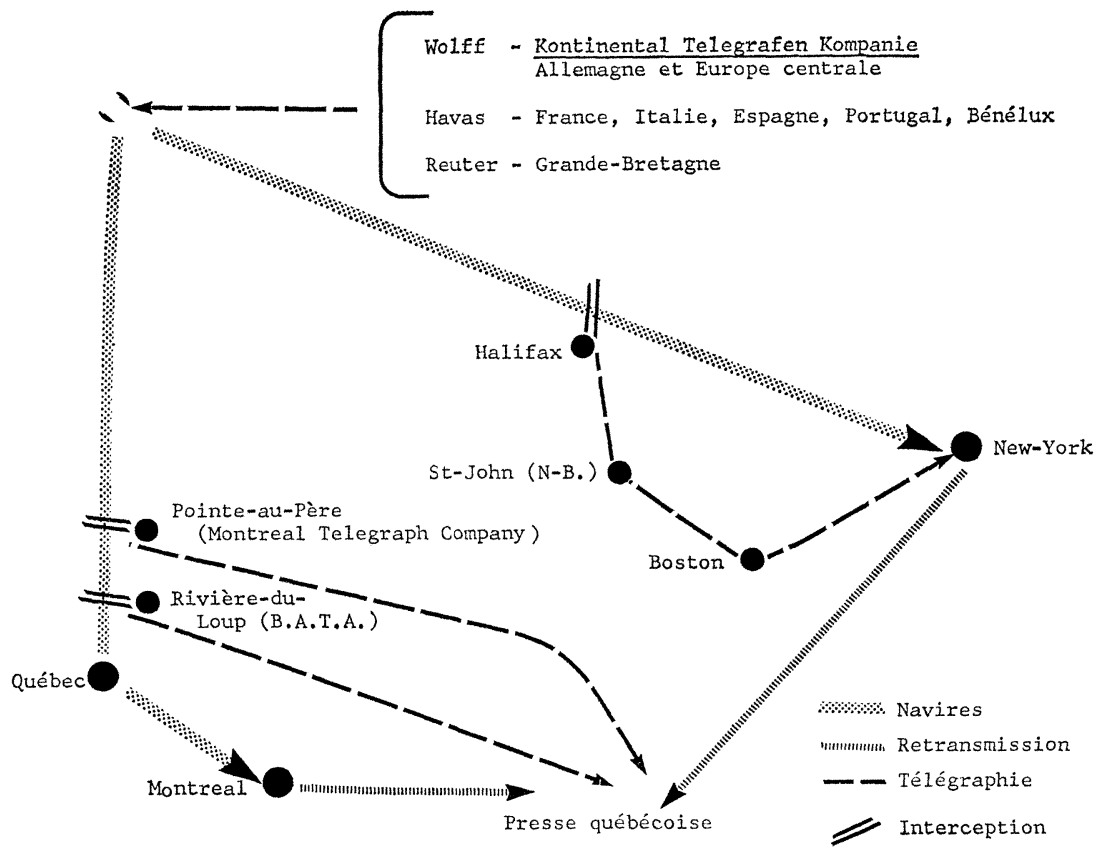

TABLEAU No 3a : Acheminement de la nouvelle européenne (1849-1856). 
tir d'Halifax, de résumés de nouvelles européennes. Au cours de 1849, pendant que se construit la ligne télégraphique unissant Halifax à Saint John (N.B.), cette association organise un "pony express" qui effectue la liaison entre Halifax et Digby. ${ }^{70}$ Un bateau à vapeur couvre la distance de Saint John à Digby en trois heures. Les nouvelles transmises de cette façon arrivent trente-cinq heures avant le bateau à vapeur Cunard. Lorsque Halifax est finalement relié en direct à New-York, les nouvelles d'Europe arrivent à New-York une cinquantaine d'heures plus vite. La transmission d'un résumé de 3000 mots à partir d'Halifax coûte $\$ 1,000.00$ à l'Associated Press. ${ }^{71}$ C'est le réseau qui est effectivement utilisé par l'Associated Press de 1849 à 1856. Mais en 1856, le réseau s'étend jusqu'à Cape Race (Terre-Neuve) et, jusqu'en 1866, des résumés de nouvelles et de dépêches européennes y sont captés puis retransmis à New-York. ${ }^{72}$

Les compagnies de télégraphe opérant au Québec tentent d'accélérer l'acheminement de la nouvelle européenne de la même façon que l'Associated Press. Vers 1854, la British American Telegraph Association établit un poste télégraphique à Rivièredu-Loup. Les vapeurs qui remontent en direction de Québec et de Montréal y font escale. De cet endroit, on télégraphie donc des dépêches et des résumés de nouvelles à Montréal. ${ }^{73} \mathrm{~A}$ la même époque, la Montreal Telegraph Company établit un poste télégraphique à Pointe-au-Père (Father Point), ${ }^{74}$ à quelques milles à l'est de Rimouski, où s'effectue le changement de pilote pour la remontée du Saint-Laurent. On en profite pour descendre les journaux européens les plus récents afin d'en télégraphier un résumé à Québec et à Montréal. La plupart des dépêches publiées dans la presse québécoise avant la pose du câble transatlantique (28 juillet 1866) sont transmises à partir de Pointeau-Père.

L'amélioration des communications télégraphiques entre le Québec et l'Europe fait couler beaucoup d'encre dans nos journaux. Les journalistes québécois manifestent un grand intérêt pour tout ce qui s'y rattache. On suit avec admiration le développement technique que constitue la pose du câble transatlantique en passant en revue les tentatives infructueuses de 1858 et de 1865 . On raconte avec force péripéties la pose réussie du

70 John Quinpool, First Things in Acadia (Halifax, 1936), 127-129.

71 Ibid., 126.

72 Ibid., 296-7.

73 John Murray, The Story of the Telegraph (Montreal, John Lovell and Son, 1905), 196.

74 Ibid., 142. 


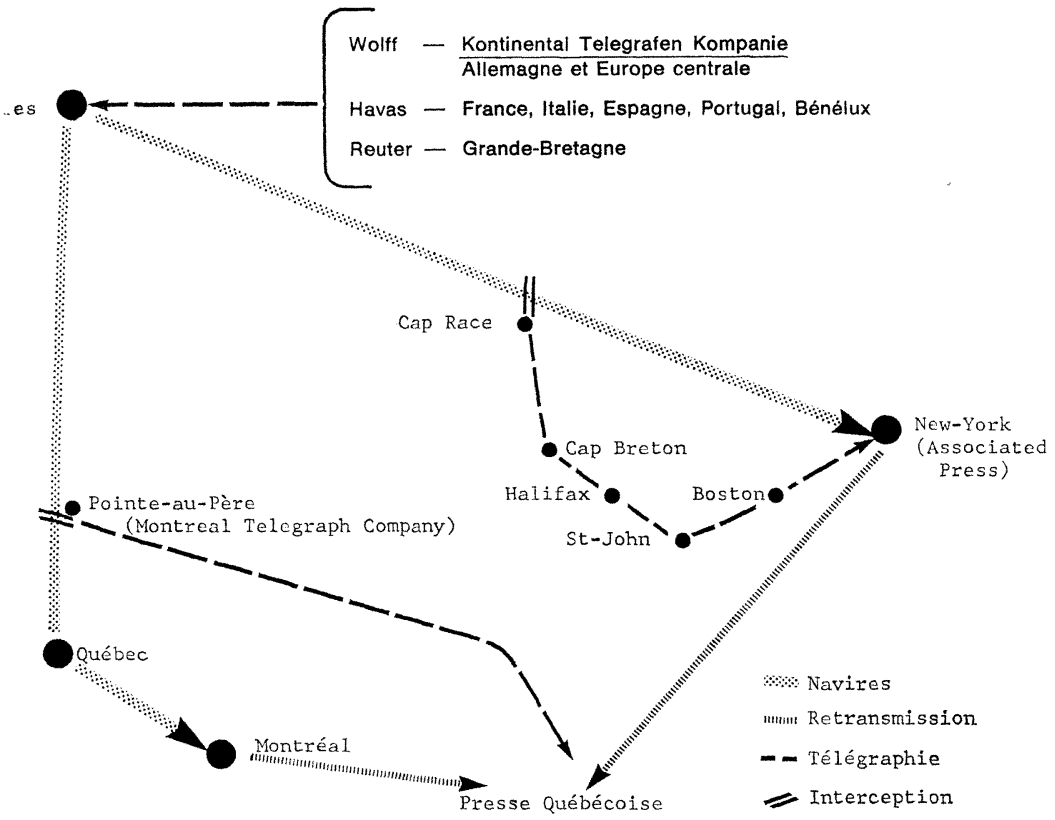


câble de 1866 et le repêchage de celui de 1865, que l'on termine. La majorité des journaux acclament le succès du 28 juillet $1866 .{ }^{75}$ Tous suivent de près les résultats obtenus, et la plupart admirent la réussite financière que représente le câble. ${ }^{76}$ La presse québécoise se montre impressionnée par ce que coûte à l'Associated Press ce service de dépêches. Le Pays est sans conteste le plus fervent admirateur du câble qui marque un net progrès dans les communications. ${ }^{77}$

Mais une opposition à cette forme d'acheminement de la nouvelle européenne ne tarde pas à se faire sentir dans les milieux journalistiques québécois. Le Pays se fait le défenseur du câble et de l'Associated Press, critiqués par le Courrier $d u$ Canada, qui s'attaque au "matérialisme" et au "modernisme" que représente la tendance toujours croissante des journaux à privilégier la dépêche comme principale source de la nouvelle. ${ }^{78}$ Tout en condamnant la curiosité du lecteur américain - curiosité "poussée jusqu'aux extrêmes limites de l'impossible" - La Minerve publie, malgré sa répugnance à le faire, des dépêches et des feuilletons pour satisfaire ses lecteurs. ${ }^{79} \mathrm{La}$ Minerve préférerait "instruire" le lecteur plutôt que de lui offrir ce qu'il désire. En apprenant à ses lecteurs que le câble est en panne, Le Courrier du Canada leur annonce que "ceux qui tiennent à avoir des nouvelles sûres, se consoleront facilement de la perte de ce mode de transmission".80 Cette sortie du Courrier agace fortement le rédacteur du Pays, qui donne la réplique en traitant ce journal d'obscurantiste. ${ }^{81}$

Après l'installation du câble, les principales nouvelles d'Europe parviennent au Québec avec une moyenne de deux jours de retard. Ces dépêches ont le désavantage cependant d'être choisies par le correspondant du Herald de New-York à Londres, le représentant attitré de l'Associated Press. ${ }^{82}$ Elles sont retransmises en anglais à la presse québécoise d'expression française par l'entremise de la Montreal Telegraph Company abonnée au

75 Le Courrier du Canada, '30 juillet 1866; Le Pays, 31 juillet 1866 ; L'Ordre, 1er août 1866.

${ }^{76}$ Le Canadien, 22 août 1866; Le Pays, 1er septembre 1866; L'Ordre, 3 septembre 1866 .

77 Le Pays, 16 août et 1er septembre 1866; 23 juillet 1870.

78 Le Courrier du Canada, 1 er août 1866.

79 La Minerve, 2 janvier 1867.

80 Le Courrier du Canada, 26 septembre 1866.

81 Le Pays, 29 septembre 1866.

82 Le Canadien, 17 août 1866. 


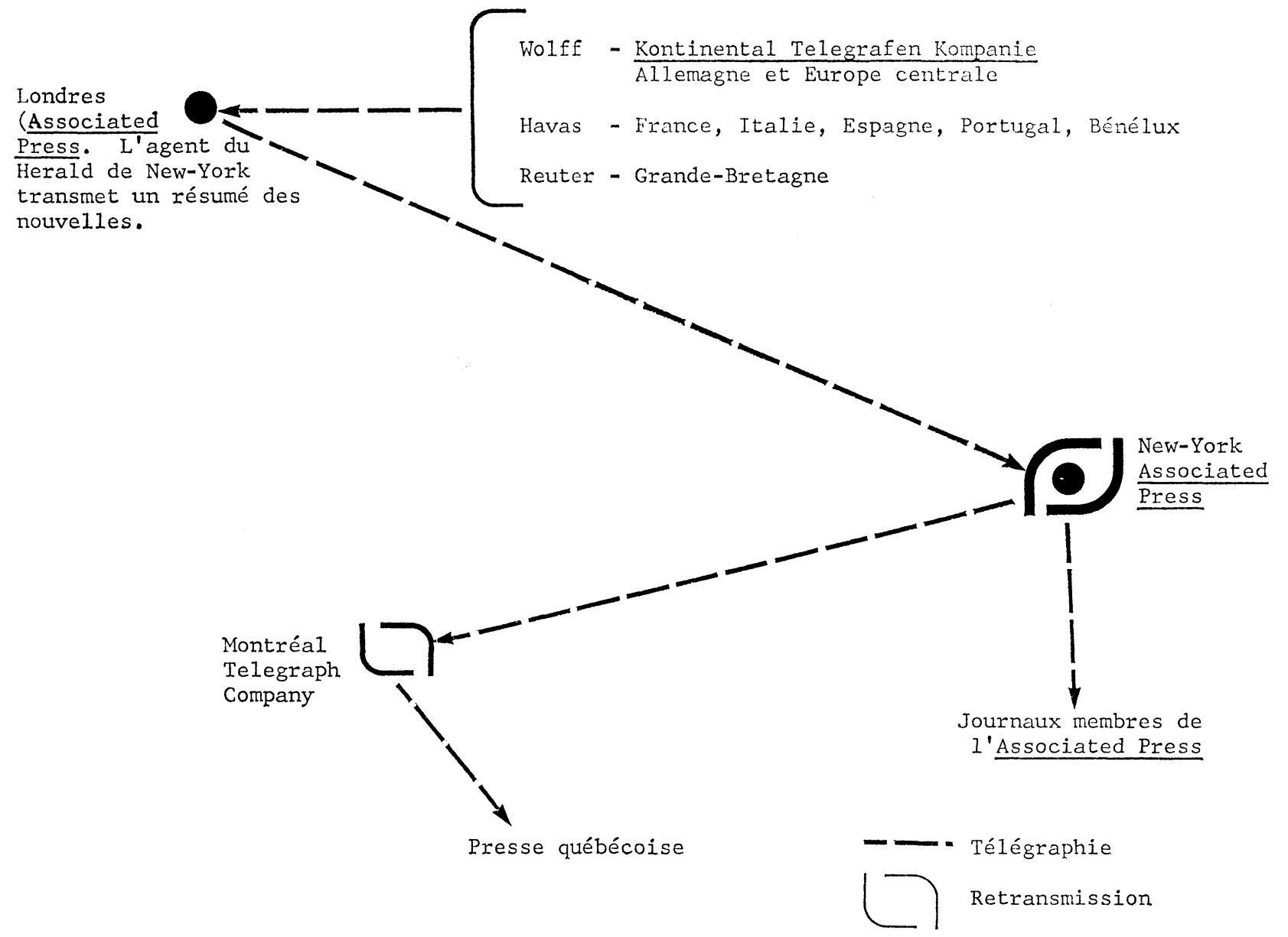

TABLEAU NO 3c: Acheminement de la nouvelle européenne (1866-1871). 
service de l'Associated Press. ${ }^{83}$ Il est inutile de revenir sur la méfiance avec laquelle la presse québécoise de langue française accueille ces dépêches qui arrivent "via Berlin et via Londres" pour ne pas dire via New-York. Le monopole de l'Associated Press n'est pas entamé par la construction d'un câble français de Brest à Saint-Pierre en 1869. Cette ligne est achetée en 1873 par l'Anglo-American Telegraph Company, qui complète la ligne en construisant le tronçon qui relie Saint-Pierre à Canso (N.E.).$^{84}$

\section{CONCLUSION}

La nouvelle européenne provient presque invariablement des mêmes sources. Celles-ci sont francophones et francophiles pour la plupart et elles affectent un très fort parti pris religieux ou politique. De plus, l'accès à la nouvelle européenne est limité, les journaux manquant de ressources financières. Rares sont ceux qui peuvent se payer de nombreux abonnements et rarissimes sont ceux qui reçoivent les dépêches acheminées par câble. Les mieux nantis sont les organes ministériels, tels La Minerve et Le Courrier $d u$ Canada. Les contrats d'impression du gouvernement leur permettent de s'offrir ce qui n'est pas à la portée des journaux de l'opposition.

Acheminée par mer, la nouvelle européenne parvient avec plus de deux semaines de retard au Québec. Cette nouvelle provient essentiellement de journaux auxquels la presse québécoise est abonnée ou avec lesquels elle échange. Quelques lettres ou chroniques viennent compléter le tout. Ce n'est qu'avec l'installation du câble (juillet 1866) que la nouvelle est acheminée en quelques jours jusqu'aux journaux québécois d'expression française. Mais le journaliste québécois se méfie de ce mode de transmission de la nouvelle européenne; il ne l'acceptera qu'à contrecœur. Il préfère l'article de fond et ne peut se résoudre à la simple énumération des faits. Il conçoit autrement la "mission" du journaliste.

${ }^{83}$ Le Journal de Trois-Rivières, 21 juillet 1870, qui s'abonne au service offert par la Montreal Telegraph Company; La Minerve, 23 juillet 1870.

$\mathbf{8 4}$ Encyclopedia Canadiana (édition de 1971), II : 154. 